\title{
Razonamiento contrafáctico, responsabilidad y culpa de la violencia contra las mujeres en la pareja: educación y medios de comunicación como factores preventivos
}

\section{Counterfactual reasoning, responsibility and blame for intimate partner violence against women: education and mass media as preventive factors}

\author{
Susana Segura \\ Departamento de Psicología Básica. Facultad de Psicología. Universidad de Málaga, España \\ Disponible online 31 de diciembre de 2012
}

\begin{abstract}
Esta investigación evalúa la percepción de las muertes por violencia contra las mujeres en la pareja. Se describen dos experimentos que analizan los efectos de la controlabilidad y la perspectiva en la causalidad percibida de estos sucesos medida a través del razonamiento contrafáctico sobre el pasado y sobre el futuro así como de las atribuciones de responsabilidad y culpa, evaluando los efectos de la empatía con una víctima en el segundo experimento. Las adscripciones de las respuestas de los participantes se centraron en factores controlables que fueron clasificados en cuatro categorías: el agresor, la víctima, las autoridades formales y la educación y los medios de comunicación. Los resultados muestran que los razonamientos contrafácticos dependen de la empatía, mientras que las atribuciones dependen de la empatía y la perspectiva. Estos resultados evidencian asociaciones entre estos procesos cognitivos, establecen factores de prevención sobre estos sucesos y permiten delimitar implicaciones específicas de este estudio para la educación y los medios de comunicación.
\end{abstract}

Palabras clave: Violencia contra las Mujeres; Educación; Medios de Comunicación; Razonamiento Contrafáctico; Responsabilidad; Culpa.

This study focused on the perception of death due to intimate partner violence against women. I describe two experiments that analyzed the effects of controllability and perspective on the perceived causality of these events measured by means of counterfactual reasoning about the past and the future as much as attributions of responsibility and blame. The effects of empathy for a victim in the second experiment were also investigated. The participants' replies focused on controllable factors which were classified into four categories: perpetrator, victim, formal authority, and education and mass media. The results show that counterfactual reasoning depends on empathy whereas attributions depend on both empathy and perspective. These results demonstrate an association between these cognitive processes and also help to establish some factors that may prevent these events. Finally, specific implications for education and the mass media are drawn from the study.

Key words: Violence against Women; Education; Mass Media; Counterfactual Reasoning; Responsibility; Blame

Correspondencia: Dra. Susana Segura. Departamento de Psicología Básica. Facultad de Psicología. Universidad de Málaga. 29071 Málaga. España. Tlf.: +34952132978. Fax: +34952132631. E-mail: s_segura@uma.es. 
La violencia contra las mujeres genera una investigación multidisciplinar cuya intención es la exploración de sus causas personales, situacionales y socioculturales (Heise, 1998). Hart y Honoré (1985) afirmaron que tanto en el derecho como en el pensamiento diario existen dos tipos de causa: la causa de hecho (cause in fact), que es el factor que provoca directamente el suceso y se establece a través del razonamiento causal, y la causa condicionante (but for cause), que es el factor que se convierte en la condición para que pueda producirse el suceso y que se establece a través del razonamiento contrafáctico.

\section{Razonamiento contrafáctico sobre el pasado y el futuro}

Este proceso cognitivo consiste en imaginar una o varias alternativas y compararlas con la situación presente y emerge, especialmente, ante resultados pasados negativos y excepcionales con objeto de aprender para el futuro. Este aprendizaje se produce por las relaciones causales que permite, ya que establecer que de no existir una alternativa imaginada la situación actual sería diferente, lleva las personas a convertir esa alternativa en la causa de la situación (para una revisión, Roese y Olson, 1995). Sin embargo, mientras que el razonamiento causal está centrado en la covariación y en factores generales, el razonamiento contrafáctico está más centrado tanto en la prevención como en eventos controlables y se aplican a factores específicos (Mandel, 2003; Mandel y Lehman, 1996; McGill y Klein, 1993; McEleney y Byrne, 2006).

Del mismo modo, las personas tienden seleccionar factores controlables tanto a nivel individual como colectivo, aunque a nivel individual se ha observado esta tendencia en los razonamientos sobre el pasado mientras que a nivel colectivo se ha observado en los razonamientos sobre el futuro. Además, a nivel colectivo, el razonamiento tiene unas cualidades diferentes ya que el aprendizaje es público porque emerge ante eventos que son aislados para una persona pero que pueden afectar a otros (para una revisión, Segura y Morris, 2005).

\section{Razonamiento contrafáctico, atribuciones de responsabili- dad y de culpa}

Este razonamiento está asociado a la responsabilidad a nivel individual pues cuando las personas perciben que tienen más responsabilidad muestran mayor arrepentimiento (Zhang, Zhou y Luo, 2009). Ambos están asociados al control de la situación pues las personas se arrepienten de las decisiones cuando tienen varias opciones (Roese y Summervile, 2005) y estas situaciones les producen mayores sentimientos de responsabilidad (Zeelenberg, Van dijk y Manstead, 1998). A nivel colectivo, la responsabilidad está asociada a la percepción de control sobre la situación y al razonamiento contrafáctico pues las personas asignan una responsabilidad vicaria por fallar en la prevención a los que tienen control sobre los que hayan causado daño a un grupo (Shultz, Jaggi y Scheleifer, 1987) y cuando las personas quieren negar responsabilidad en un grupo sobre unos sucesos, niegan el control negando conocimiento de la situa- ción e imposibilitando que exista tanto posibilidad de prevención como de enlace causal entre ellos y los sucesos (Markman y Tetlock, 2000).

Igualmente, está asociado a atribuciones de culpa a través del efecto de orden temporal, que consiste en seleccionar el último antecedente en una secuencia de sucesos independientes previos a un evento cuando se realizan razonamientos contrafácticos. En sucesiones de dos eventos, las personas piensan que el individuo que ha producido el segundo se siente más culpable que el individuo que ha producido el primero y el primero culpará más al segundo (Byrne, Segura, Culhane, Tasso y Berrocal, 2000; Miller y Gunasegaram, 1990). A diferencia de los estudios sobre la responsabilidad, en estos estudios, los investigadores no evaluaron la culpa desde la perspectiva propia de los participantes sino desde la perspectiva de los demás o perspectiva ajena, pues tenían que juzgar imaginando como se sentían los individuos que tomaban parte en los sucesos. Esta perspectiva requiere de la empatía, pues supone imaginar los sentimientos de los demás, pero habría que distinguir entre imaginarse los sentimientos de uno o varios individuos que toman parte en los eventos e imaginarlos desde la posición de una persona concreta. Además, la empatía con una persona en particular influye también en otros procesos cognitivos: a mayor empatía con una víctima, se produce una mayor simpatía hacia ella así como la intención de entregarle una mayor compensación (Macrae y Milne, 1992).

\section{Atribuciones de responsabilidad y de culpa a nivel indivi- dual y colectivo}

La selección de factores controlables evidencia una búsqueda mental de las causas de los hechos que está relacionada con las personas responsables o culpables de producir el suceso o de fallar en su prevención. Shaver (1985) propuso la Teoría de la Atribución Defensiva que consta de tres etapas: primero es necesario que la persona sea considerada la causa del evento; segundo, responsable y finalmente, culpable. Las características de cada atribución hasta llegar a la culpa se suceden en un continuo. La atribución de causalidad debe realizarse sobre un factor humano que sea una fuente identificable de la acción u omisión a nivel individual o grupal y se perciba que ha podido prever el suceso. Para atribuirle la responsabilidad, es necesario que su conducta no esté justificada por la situación y que ha operado en condiciones de libertad de elección, es decir, que ha llevado a cabo un acto controlable y consciente. Para que se le atribuya la culpa, es necesario además que la valencia del suceso sea negativa y se perciba que ha habido intención de conseguir ese resultado negativo, por lo que está relacionado con el merecimiento de un castigo.

Otras investigaciones señalan que para que se atribuya responsabilidad a alguien deben darse las siguientes condiciones: la persona debe considerarse necesaria y no solo suficiente como causa de un acontecimiento negativo (Shultz, Schleifer y Altman, 1981), tener control de la situación (Schlenker, Britt, 
Pennington, Murphy y Doherty, 1994), romper las normas sociales (Devos-Comby y Devos, 2001) y que conlleve graves consecuencias (Chaikin y Darley, 1973). Además, a más graves sean las consecuencias percibidas, mayor responsabilidad se le atribuye a la persona a la que se le asocia (Robbennolt, 2000); mientras que para atribuir la culpa a alguien es necesario que también se perciba una intención de hacer daño (Lagnado y Channon, 2008).

Respecto a la responsabilidad colectiva, en el ámbito de las organizaciones, la responsabilidad y la culpa han sido entendidas como sinónimos y se atribuye al líder o representante por equivocarse y por negligencia (Hamilton, 1978a; Hamilton, 1978b; Hamilton, 1986), debido a la tendencia a creer que tiene control de la situación (Meindl, Ehrlich y Dukerich, 1985) y esto sucede en distintas culturas (Zemba, Young y Morris, 2006). En el ámbito penal, han sido entendidas del mismo modo en un estudio centrado en el asesinato múltiple llevado a cabo en el instituto Columbine en los EE.UU., en el que se atribuye la responsabilidad por la acción a los agresores, que eran dos adolescentes, y por omisión de conductas para prevenir los sucesos a los padres (Lickel, Schmader y Hamilton, 2003).

\section{Atribuciones de responsabilidad y de culpa en la violencia contra las mujeres en la pareja}

Relacionada con el modelo de Shaver (1985), la Teoría de la Creencia en un Mundo Justo (Lerner, 1980), que defiende que las personas creen que a los individuos buenos les ocurren cosas buenas y a los individuos malos les ocurren cosas malas, explica que las personas perciben que viven en un mundo controlable y equitativo en una necesidad defensiva de protegerse del temor a que les ocurran hechos sin motivo. Esta teoría ha sido propuesta como explicación en una parte de las investigaciones existentes sobre la atribución de la culpa a las víctimas, pues implica que se merecen lo que les sucede (para una revisión, Furnham, 2003). En el caso de las víctimas de este tipo de violencia, esas creencias y el sexismo hostil afloran para culpar a la víctima cuando no se menciona la causa de la agresión debido a la ambigüedad de la situación. Además, las creencias sexistas influyen independientemente de las otras (Valor-Segura, Expósito y Moya, 2008, 2011). Efectivamente, las creencias en un mundo justo están motivadas por la incertidumbre personal sobre cómo van a actuar en el futuro (Ball y Van Den Bos, 2012). Y las creencias sexistas reflejan una normalización de la violencia, de tal manera que a más benevolente se sea con el sexismo, más probabilidad existe de que se perciba que el hombre va a actuar de modo violento cuando se sienta amenazado (Herrera, Expósito, Moya y Houston, 2012). Estudios cualitativos y reflexiones teóricas defienden que esa normalización hace que las mujeres sean vistas como cómplices de su propia victimización porque el peso de la responsabilidad recae sobre ellas, acaban interiorizando esas normas y culpándose a sí mismas (Thapar-Björkert y Morgan, 2010), siendo esta auto culpa exacerbada por la justicia (Koss, 2000).
Mientras que para que las personas manifiesten intención de mediación y denuncia necesitan percibir responsabilidad individual, requiriendo además percibir gravedad en los hechos para la segunda acción (Gracia, García y Lila, 2009).

Por último, otros estudios centrados en aspectos sociales o personales muestran diferencias en cuanto al género, al consumo de alcohol o a ambos factores pero con resultados poco concluyentes sobre las atribuciones de responsabilidad y culpa (por ejemplo, véase, Stewart y Maddren, 1997; Brigitte, y Knowles, 2000).

\section{El presente estudio}

La revisión de la literatura muestra una ausencia de estudios que exploren la causalidad condicionante percibida de la violencia contra las mujeres, que cuando llevan a la muerte pueden ser sucesos determinantes de razonamientos contrafácticos pues son negativos, graves, excepcionales, rompen las normas sociales y se repiten a nivel colectivo, ya que pueden sucederles a otra persona de la comunidad por parte del mismo individuo o de otro individuo. Por lo tanto, el objetivo general del presente estudio es analizar los procesos cognitivos que subyacen a la percepción de la muerte por violencia contra las mujeres.

\section{Experimento 1}

Como se ha señalado, no se han evaluado los razonamientos sobre el pasado y futuro conjuntamente, ni con las atribuciones de responsabilidad y culpa, estando estas variables relacionadas entre sí por con la controlabilidad percibida tanto a nivel individual como colectivo. Asimismo, a estos dos niveles no se han evaluado simultáneamente con estos razonamientos las atribuciones de responsabilidad y culpa, habiendo sido analizadas además desde una única perspectiva. Consiguientemente, la hipótesis de este primer experimento consiste en evaluar los efectos de la controlabilidad en el razonamiento contrafáctico sobre el pasado (RCP) y el razonamiento contrafáctico sobre el futuro (RCF) así como los efectos tanto de la controlabilidad como de la perspectiva en las atribuciones de responsabilidad (AR) y las atribuciones de culpa (AC) sobre las muertes por violencia contra las mujeres.

\section{Método}

Participantes. Los participantes fueron 157 mujeres y 47 hombres, estudiantes de la Universidad de Málaga, España, que fueron asignados equivalentemente a cada condición experimental.

Materiales. Se establecieron dos condiciones respecto a la perspectiva de las atribuciones: "perspectiva propia" (donde los participantes deben realizar una atribución externa de responsabilidad y culpa desde su perspectiva) y a la condición "perspectiva ajena" (donde los participantes deben determinar sentimientos como consecuencia de una atribución interna de responsabilidad y culpa desde la perspectiva de otra u otras personas) del mismo modo que en los estudios previos (Byrne et 
al., 2000; Miller y Gunasegaram, 1990). Asimismo, tal y como fue llevado a cabo en el estudio sobre el instituto Columbine (Lickel et al., 2003), las respuestas fueron clasificadas en categorías en función de los factores mencionados por los participantes: agresor, víctima, autoridades formales (gobierno, policías, jueces y jurados) y educación y medios de comunicación (educadores, responsables de los medios de comunicación...). Estas categorías constituyen dos tipos diferenciados de factores controlables o humanos: los dos primeros corresponden a los individuos que participan en los sucesos de forma personal y los dos últimos, a los colectivos o grupos que participan en los sucesos como dos tipos de representantes de toda la comunidad. Por lo tanto, para realizar un análisis más explicativo, las respuestas fueron además reagrupadas en dos categorías: individuos y colectivos. Cada categoría es independiente y los participantes pueden mencionar más de una, por lo que no coincide el número de participantes con el número de observaciones.

La evaluación se realizó a través de preguntas abiertas utilizando el término malos tratos, como aparecen en los instrumentos diseñados por Gracia et al. (2009). En la condición experimental "perspectiva propia" fueron las siguientes:

"¿Crees que las muertes por malos tratos a las mujeres podrían haber sido evitadas?

¿Cómo? Por ejemplo,...

¿Quién crees que es o son los responsables?

¿Quién crees que es o son los culpables?

¿Qué medidas crees que se podrían tomar en el futuro para evitar este tipo de muertes?

Por ejemplo,..."

En la condición experimental "perspectiva ajena" las preguntas sobre las atribuciones fueron substituidas por las siguientes:

"¿Quién crees que se siente o se sienten responsables?

¿Quién crees que se siente o se sienten culpables?"

Procedimiento. Cumpliendo las normas éticas pertinentes, en el horario habitual de clases, sin que reportara ninguna compensación a los colaboradores en la solicitud de participación ni a los participantes y sin límite de tiempo, se repartió a estos participantes un cuadernillo con las siguientes instrucciones: "Gracias por tomar parte en este experimento. Te pedimos que leas con cuidado y que contestes a unas sencillas preguntas que aparecen en la siguiente página. Por favor, presta mucha atención y no cambies tu respuesta una vez la hayas escrito. Recuerda que no se trata de evaluar tus conocimientos y que tu participación es anónima".

\section{Resultados}

Las proporciones de las adscripciones de los participantes a las categorías individuos y colectivos para las dos condiciones experimentales se presentan en la figura 1 .

RCP Y RCF. Se llevaron a cabo análisis de contrates de proporciones de categorías no relacionadas. En la condición "perspectiva propia", los participantes respondieron que las muertes se podrían haber evitado $(95 \%$ versus $5 \%, n=102$, $z=20.85, p<.05)$. El RCP se centró menos en los individuos que en los colectivos ( $18 \%$ versus $82 \%, n=149, z=5.71, p<$ $.02)$, menos en el agresor que en la víctima ( $1 \%$ versus $17 \%$, $n=149, \mathrm{z}=-4.97, p<.02)$ e igualmente en los dos colectivos ( $46 \%$ versus $36 \%, n=149, z=1.35, p=.08)$. El RCF se centró menos en los individuos que en los colectivos ( $3 \%$ versus $97 \%$, $n=149, z=-33.63, p<.02)$ e igualmente en el agresor que en la víctima ( $1 \%$ versus $2 \%, n=149, z=-0.70, p=.24)$ y en $\operatorname{los}$ dos colectivos ( $46 \%$ versus $51 \%, n=149, z=-0.62, p=.26$ ).

En la condición "perspectiva ajena", los participantes respondieron que las muertes se podrían haber evitado $(95 \%$ versus $5 \%, n=102, z=20.85, p<.05)$. El RCP se centró menos en los individuos que en los colectivos ( $29 \%$ versus $71 \%, n=$ $135, z=-5.37, p<.02)$, menos en el agresor que en la víctima ( $2 \%$ versus $27 \%, n=135, z=-6.09, p<.02)$ e igualmente en los dos colectivos (35\% versus 36\%, $n=135, z=-0.13, p$ $=.44)$. El RCF se centró menos en los individuos que en los colectivos ( $9 \%$ versus $91 \%, n=139, z=-16.89, p<.02$ ), menos en el agresor que en la víctima ( $1 \%$ versus $8 \%, n=139, z=$ $-2.82, p<.02)$ e igualmente en los dos colectivos $(43 \%$ versus $48 \%, n=139, z=-0.61, p=.26)$.

AR y AC. Se llevaron a cabo análisis estadísticos equivalentes a los anteriores. En la condición "perspectiva propia", la AR se centró menos en los individuos que en los colectivos (39\% versus $61 \%, n=174, z=-2.97, p<.01)$, más en el agresor que en la víctima ( $26 \%$ versus $13 \%, n=174, z=2.80, p<.01)$ e igualmente en los dos colectivos ( $25 \%$ versus $36 \%, n=174, z$ $=-1.87, p=.03)$. La AC se centró igualmente en los individuos que en los colectivos ( $52 \%$ versus $48 \%, n=157, z=0.50, p=$ $.30)$, más en el agresor que en la víctima (37\% versus $15 \%, n=$ $157, z=4.01, p<.01)$ y menos en las autoridades formales que en la educación y medios de comunicación (17\% versus $31 \%, n$ $=157, z=-2.58, p<.01)$.

En la condición "perspectiva ajena", la AR se centró más en los individuos que en los colectivos (59\% versus $41 \%, \mathrm{n}=134$, $\mathrm{z}=2.11, \mathrm{p}<.01)$, menos en el agresor que en la víctima $(19 \%$ versus $40 \%, n=134, z=-3.29, p<.01)$ y menos en las autoridades formales que en la educación y medios de comunicación $(10 \%$ versus $31 \%, n=134, z=-4.01, p<.01)$. La AC se centró más en los individuos que en los colectivos ( $76 \%$ versus $24 \%, n$ $=120, z=6.66, p<.01)$, menos en el agresor que en la víctima $(26 \%$ versus $50 \%, n=120, z=-3.13, p<.01)$ y menos en las autoridades formales que en la educación y medios de comunicación ( $2 \%$ versus $22 \%, n=120, z=-4.89, p<.01)$.

Entre condiciones experimentales se llevaron a cabo los mismos análisis estadísticos pero para muestras independientes. En cuanto a la AR, se centró menos en la condición "perspectiva propia" que en la condición "perspectiva ajena" en los individuos $(39 \%, n=174$, versus $59 \%, n=134, z=-3.48, p<$ $.01)$, encontrándose lo contrario para los colectivos $(61 \%, n=$ 174 , versus $41 \%, n=134, z=3.48, p<.01)$. No se encontraron diferencias significativas respecto al agresor $(26 \%, n=174$, 
versus $19 \%, n=134, z=1.44, p=.07)$, se centró menos en la víctima $(13 \%, n=174$, versus $40 \%, n=134, z=5.44, p<.01)$, más en las autoridades formales $(25 \%, n=174$, versus $10 \%, n$ $=134, z=3.36, p<.01)$ y no se encontraron diferencias significativas respecto a la educación y los medios de comunicación $(36 \%, n=174$, versus $31 \%, n=134, z=0.92, p=.17)$. En cuanto a la AC, se centró menos en la condición "perspectiva propia" en los individuos $(52 \%, n=157$, versus $76 \%, n=120, z$ $=-4.08, p<.01)$, encontrándose lo contrario para los colectivos ( $48 \%, n=157$, versus $24 \%, n=120, z=4.08, p<.01)$. No se encontraron diferencias significativas respecto al agresor $(37 \%$, $n=157$, versus $26 \%, n=120, z=1.94, p=.02)$, se centró menos en la víctima $(15 \%, n=157$, versus $50 \%, n=120, z$ $=6.28, p<.01)$, más en las autoridades formales $(17 \%, n=$ 157 , versus $2 \%, n=120, z=4.03, p<.01)$ y no se encontraron diferencias significativas respecto a la educación y los medios de comunicación $(31 \%, n=157$, versus $22 \%, n=120, z=1.67$, $p=.04)$.

\section{Figura 1}

Proporciones de las adscripciones de los participantes a las categorías de respuesta agrupadas para las condiciones experimentales clasificadas por las variables dependientes (experimento 1).

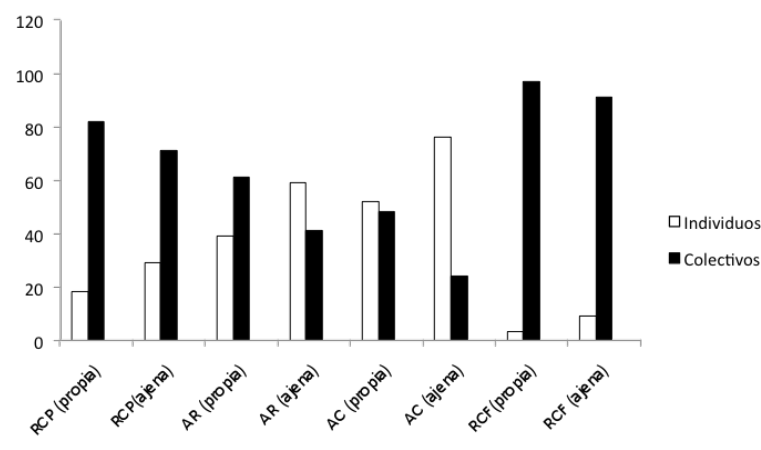

Experimento 2

Debido a las diferencias entre la perspectiva y la empatía expuestas en la introducción, la hipótesis de este segundo experimento es evaluar las mismas variables del primero pero posicionando a los participantes en la situación de una víctima.

\section{Método}

Participantes. Los participantes fueron 125 mujeres y 45 hombres, estudiantes de la Universidad de Málaga, España, que fueron asignados equivalentemente a cada condición experimental.

Materiales. Las preguntas para ambas condiciones experimentales fueron idénticas a las del experimento 1 con la diferencia de que delante de esas preguntas recibían el siguiente enunciado: "Imagina que eres una mujer víctima de malos tratos por parte de tu pareja sentimental".

Procedimiento. Fue idéntico al utilizado para el experimento 1 a excepción de la parte de las instrucciones en la que, para ajustarlas al enunciado referido, la frase encabezada por la demanda para colaborar en la investigación fue substituida por: "Te pedimos que leas con cuidado lo que aparece en la siguiente página y que contestes a unas sencillas preguntas...”.

\section{Resultados}

Las proporciones de las adscripciones de los participantes a cada categoría se presentan del mismo modo que en el experimento 1 en la figura 2. Los análisis estadísticos que se llevaron a cabo fueron equivalentes a los del experimento 1 .

RCP y RCF. En la condición "perspectiva propia” los participantes respondieron que las muertes se podrían haber evitado (98\% versus $2 \%, n=85, z=31.61, p<.05)$. El RCP se centró igualmente en los individuos que en los colectivos (43\% versus $57 \%, n=106, z=-1.45, p=.07)$, menos en el agresor que en la víctima ( $3 \%$ versus $40 \%, n=106, z=-7.03, p<.02$ ) e igualmente en los dos colectivos ( $33 \%$ versus $24 \%, n=106, z$ $=1.23, p=.10)$. El RCF se centró menos en los individuos que en los colectivos ( $6 \%$ versus $94 \%, n=117, z=-20.04, p<.02)$, igualmente en el agresor que en la víctima (1\% versus 5\%, $n$ $=117, z=-1.79, p=.03)$ y en los dos colectivos $(39 \%$ versus $55 \%, n=117, z=-1.81, p=.03)$.

En la condición "perspectiva ajena", los participantes respondieron que las muertes se podrían haber evitado ( $98 \%$ versus $2 \%, n=85, z=31.610, p<.05)$. El RCP se centró igualmente en los individuos que en los colectivos ( $40 \%$ versus $60 \%, n=$ $118, z=-1.846, p=.03)$, menos en el agresor que en la víctima (3\% versus $37 \%, n=118, z=-6.92, p<.02)$ e igualmente en los dos colectivos ( $35 \%$ versus $25 \%, n=118, z=1.41, p=.07$ ). El RCF se centró menos en los individuos que en los colectivos $(10 \%$ versus $90 \%, n=119, z=-14.54, p<.02)$. No se llevaron a cabo contrastes entre las proporciones de respuestas centradas en el agresor por encontrarse valor 0 y se centró igualmente en los dos colectivos ( $48 \%$ versus $42 \%, n=119, z=0.69, p=.24$ ).

AR y AC. En la condición "perspectiva propia", la AR se centró igualmente en los individuos que en los colectivos (56\% versus $44 \%, n=123, z=-1.34, p=.09)$, más en el agresor que en la víctima ( $40 \%$ versus $16 \%, n=123, z=3.75, p<.01)$ y menos en las autoridades formales que en la educación y medios de comunicación ( $11 \%$ versus $33 \%, n=123, z=-3.89, p<.01)$. La AC se centró más en los individuos que en los colectivos $(70 \%$ versus $30 \%, n=119, z=4.76, p<.01)$, más en el agresor que en la víctima ( $59 \%$ versus $11 \%, n=119, z=7.64, p<.01)$ y menos en las autoridades formales que en la educación y medios de comunicación ( $5 \%$ versus $25 \%, n=119, z=-4.27, p<.01$ ).

En la condición "perspectiva ajena”, la AR se centró más en los individuos que en los colectivos ( $62 \%$ versus $38 \%, n=99$, $z=2.46, p<.01)$, menos en el agresor que en la víctima $(18 \%$ versus $44 \%, n=99, z=-3.48, p<.01)$ y menos en las autoridades formales que en la educación y los medios de comunicación ( $7 \%$ versus $31 \%, n=99, z=-4.20, p<.01)$. La AC se centró más en los individuos que en los colectivos (80\% versus $20 \%, n=111, z=7.90, p<.01)$, menos en el agresor que en la víctima $(19 \%$ versus $61 \%, n=111, z=-5.60, p<.01)$ y menos 
en las autoridades formales que en la educación y los medios de comunicación ( $1 \%$ versus $19 \%, n=111, z=-4.63, p<.01)$.

Entre condiciones experimentales, en cuanto a la AR, no se encontraron diferencias significativas respecto a los individuos (56\%, $n=123$, versus $62 \%, n=99, z=-0.90, p=.18)$, ni los colectivos $(44 \%, n=123$, versus $38 \%, n=99, z=0.90, p=$ .18), se centró más en la condición "perspectiva propia" que en la condición "perspectiva ajena" en el agresor $(40 \%, \mathrm{n}=123$, versus $18 \%, \mathrm{n}=99, \mathrm{z}=3.59, \mathrm{p}<.01)$ y menos en la víctima $(16 \%, \mathrm{n}=123$, versus $44 \%, n=99, z=-4.59, p<.01)$, tampoco respecto a las autoridades formales $(11 \%, n=123$, versus $7 \%$, $n=99, z=1.02, p=.15)$ ni a la educación y los medios de comunicación $(33 \%, \mathrm{n}=123$, versus $31 \%, n=99, z=0.31, p=$ .37). En cuanto a la AC, no se encontraron diferencias significativas respecto a los individuos $(70 \%, n=119$, versus $80 \%, n$ $=111, z=-1.74, p=.04)$, ni los colectivos $(30 \%, n=119$, versus $20 \%, n=111, z=1.74, p=.04)$, se centró más en la condición "perspectiva propia" en el agresor $(59 \%, n=119$, versus $19 \%$, $n=111, z=6.19, p<.01)$ y menos en la víctima $(11 \%, n=119$, versus $61 \%, n=99, z=-7.93, p<.01)$, tampoco respecto a las autoridades formales $(5 \%, n=119$, versus $1 \%, n=99, z=1.75$, $p=.04)$ ni a la educación y los medios de comunicación (25\%, $n=119$, versus $19 \%, n=111, z=1.09, p=.13$ ).

\section{Figura 2}

Proporciones de las adscripciones de los participantes a las categorías de respuesta agrupadas para las condiciones experimentales clasificadas por las variables dependientes (experimento 2).

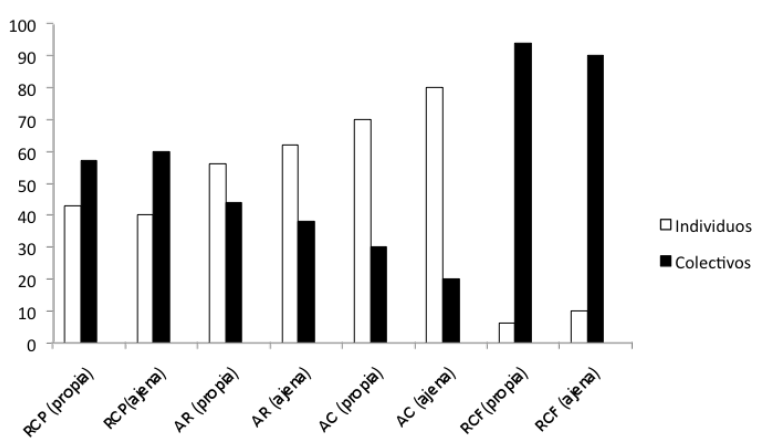

\section{Discusión}

Los resultados muestran que respecto a las dos formas de razonamiento contrafáctico, los participantes se centraron más en los dos colectivos que en los individuos y en los dos por igual, excepto en el experimento 2 en los razonamientos sobre el pasado, en el que se centraron en zlos individuos, en concreto, en la víctima. Se encontró el mismo patrón de respuesta en las dos condiciones experimentales, que fueron diseñadas únicamente para evaluar las hipótesis exploratorias para las atribuciones, lo que demuestra que los materiales son adecuados para este análisis. Congruentemente con los estudios previos, las personas perciben que estos sucesos son evitables y señalan los factores específicos controlables de los que depende su prevención (Mandel, 2003; Mandel y Lehman, 1996; McGill y Klein, 1993; McEleney y Byrne, 2006), por lo que los consideran las causas condicionantes de los hechos. Y muestran efectos de controlabilidad a nivel colectivo pues se centran en los representantes de la comunidad respecto al futuro (para una revisión, Segura y Morris, 2005), descubriendo además los mismos efectos sobre los mismos colectivos respecto al pasado en situación de no empatía, lo que indica que existe un aprendizaje grupal a través de la identificación de esas causas condicionantes.

Respecto a la situación de empatía, los resultados se explican porque estas muertes no son hechos puntuales representados en pruebas de laboratorio como los explorados en experimentos previos (Macrae y Milne, 1992) sino el final o el resultado de una sucesión de conductas que, imaginadas desde la posición de la víctima, hace que se perciba como no excepcional y, por tanto, previsible, lo que les lleva a percibirla como controlable y evitable. Esta idea se apoya en el hecho de que las personas esperan que las víctimas de esta violencia regulen las conductas de los agresores (Thapar-Björkert y Morgan, 2010) y además cuando las personas se enfrentan a su muerte realizan atribuciones de control como mecanismo defensivo (Willis, Tapia-V y Martinez, 2011). Sin embargo, en lo que respecta al futuro, antes del encuentro con el agresor y del comienzo de las conductas violentas, la víctima aún no puede tener control sobre la situación pues no tiene información, no se percibe que pueda actuar de otro modo y la prevención recae en aquellos que sí pueden cambiar esas conductas, que son lo mismos que se perciben en la situación de no empatía.

Los resultados respecto a las dos atribuciones muestran que en la condición de "perspectiva ajena" y en los dos experimentos, los participantes se centraron en los individuos, en concreto, en la víctima. Para la condición de "perspectiva propia" se encontró este patrón de respuesta respecto a la culpa en el experimento 2 , se centraron más en los colectivos y en los dos por igual respecto a la responsabilidad en el experimento $1, \mathrm{y}$ en el agresor y la educación y los medios de comunicación respecto a la culpa en el experimento 1 y respecto a la responsabilidad en el experimento 2. Entre condiciones experimentales, el patrón de respuesta fue el mismo para las dos atribuciones en el experimento 1 (en la "perspectiva propia", menos en los individuos y más en los colectivos que en la "perspectiva ajena") y en el experimento 2 (no hubo diferencias entre condiciones experimentales).

Respecto a la "perspectiva ajena", los resultados muestran que cuando los participantes han tenido que imaginarse en la situación de otro u otros han preferido imaginarse en el lugar de la víctima y se auto culpan, proceso este último que efectivamente ocurre a las víctimas de estos sucesos (por ejemplo, véase Andrews y Brewin, 1990). De acuerdo con el estudio de Herrera et al. (2012), estos resultados manifiestan una normalización de la violencia, pero además es una evidencia experimental de las conclusiones de Thapar-Björkert y Morgan (2010) que sostienen que esta normalización lleva a las personas a percibir que 
las mujeres interiorizan esas normas aunque demostrando que no es debido a la atribución de la responsabilidad.

Respecto a la "perspectiva propia", los resultados sobre la responsabilidad coinciden con los del estudio sobre el instituto Columbine (Lickel et al., 2003) que fueron atribuciones realizadas en una situación de no empatía, al atribuirla a las autoridades y a la educación, representados por los padres. Y explica el significativo debate entre los profesionales de las ciencias de la comunicación y en la sociedad acerca de la influencia de los medios de comunicación en este tipo de sucesos (Hoerl, 2002).

Al mismo tiempo, congruentemente con la teoría de Shaver (1985), las atribuciones de responsabilidad coinciden con las respuestas sobre los razonamientos en la misma situación. Sin embargo, respecto a la culpa, aunque señalan más específicamente a la educación y los medios de comunicación también señalan al agresor, por lo que no se puede confirmar que sea el último paso en estas atribuciones. Estas diferencias entre atribuciones, que también se observan en situaciones de empatía, indican que la atribución de la culpa en estos casos, está determinada por más factores que la percepción de intención de daño y que pueden ser las creencias en un mundo justo. Pero aunque la atribución de la culpa a las víctimas no aparece en aquellos que no tienen esas creencias (Van Den Bos y Mass, 2009), su aparición produce respuestas dispares de atribución de la culpa, ayuda o desacreditación dependiendo de la percepción de las características personales y conductuales de la víctima (Haynes y Olson, 2006). La dificultad para explicar estos datos procede de las limitaciones de este estudio por no haber evaluado estas variables y por la ausencia de utilización de materiales complementarios que podrán determinar futuras investigaciones.

Respecto al idéntico patrón de respuesta entre condiciones para las dos atribuciones en las categorías individuos y colectivos, los resultados determinan definitivamente las similitudes entre las atribuciones de responsabilidad y las de culpa a nivel colectivo. Además, son coherentes entre sí: en situación de no empatía, en la condición experimental que requiere imaginar la perspectiva de otro u otros, las personas se centran en la víctima, por lo que cuando están en situación de empatía con ella no hay diferencias entre los resultados en las dos condiciones experimentales.

Por último, tomados todos los datos en su conjunto, los resultados muestran que el factor que más han mencionado los participantes para los razonamientos y las atribuciones desde su perspectiva ha sido la educación y los medios de comunicación.

\section{Implicaciones para la educación y los medios de comuni- cación}

La trascendencia de la educación sobre la prevención de la violencia contra la mujer en distintas áreas es bien conocida (por ejemplo, véase Leal González y Arconada Melero, 2011). En la investigación en psicología aumentan las investigaciones sobre las condiciones en las que los medios de comunicación favorecen esta violencia (para una revisión, King, 2010).
Pero además, recientemente, las ciencias de la comunicación han comenzado a reflexionar sobre sus funciones a través de la transmisión y el tratamiento de la información, incluyendo la importancia de consultar a expertos y de llevarlo a cabo desde la empatía (Fernández Arribas, 2011). Este estudio descubre que la perspectiva o el enfoque que se le confiera a la información efectivamente va a influir en la percepción de los hechos y que, en concreto, la utilización de la empatía, respecto a la localización de la causa condicionante puede ser contraproducente si se refiere al pasado y respecto a las atribuciones de responsabilidad y culpa, en todas las perspectivas o situaciones analizadas excepto en una. Además, pone de relieve que el aprendizaje colectivo se produce cuando se hace público y ellos son los que llevan a cabo esta labor, en muchas ocasiones, a través de los representantes de las autoridades formales que aparecen en estos medios e influyendo, a su vez, en estos representantes. Finalmente, este estudio muestra que las personas imaginan situaciones alternativas para la prevención que pueden ser mostradas a través de estos medios pero enfocadas a dar ayuda a las víctimas, como la denuncia o la mediación, y que ejemplifiquen la igualdad de género desde la perspectiva adecuada con objeto de evitar atribuirles la culpa, la normalización de la violencia contra las mujeres y su internalización por parte de ellas; favoreciendo el apoyo a nivel individual y colectivo. En suma, esta investigación abre una puerta a nuevos descubrimientos en la psicología para la prevención de la violencia contra las mujeres.

\section{Referencias}

1. Andrews, B. y Brewin, C. (1990). Attributions of blame for marital violence: A study of antecedents and consequences. Journal of marriage and the family, 52, 757-767. http:// dx.doi.org/10.2307/352940

2. Ball, M. y Van Den Bos, K (2012). Blaming for a better future: future orientation and associated intolerance of personal uncertainty lead to harsher reactions toward innocent victims. Personality and Social Psychology, 38, 835-844. http://dx.doi.org/10.1177/0146167212442970

3. Brigitte, L. y Knowles, A. (2000). Community attitudes to domestic violence: Attributions of responsibility and suggested punishments related to alcohol consumption and level of violence. Psychiatry, Psychology and Law, 7, 51-58. http://dx.doi.org/10.1080/13218710009524970

4. Byrne, R. M. J., Segura, S., Culhane, R., Tasso, A. y Berrocal, P. (2000). The temporality effect in counterfactual thinking about what might have been. Memory \& Cognition, 28, 264-281. http://dx.doi.org/10.3758/BF03213805

5. Chaikin, A. L. y Darley, J. M. (1973). Victim or perpetrator?: Defensive attribution of responsibility and the need for order and justice. Journal of Personality and Social Psychology, 25, 268-275. http://dx.doi.org/10.1037/h0033948

6. Devos-Comby, L. y Devos, T. (2001). Social norms, social value, and judgments of responsibility. Swiss Journal of Psychology 60, 35-46. http://dx.doi.org/10.1024//1421- 
$\underline{0185.60 .1 .35}$

7. Fernández Arribas, J. (2011). Cómo informar sobre violencia contra la mujer en las relaciones de pareja. Madrid: Centro Reina Sofía.

8. Furnham, A. (2003). Belief in a just world: research progress over the past decade. Personality and Individual Differences, 34, 795-815. http://dx.doi.org/10.1016/S01918869(02)00072-7

9. Gracia, E., García, F. Lila, M. (2009). Public responses to intimate partner violence against women: The influence of perceived severity and personal responsibility. The Spanish Journal of Psychology, 12, 648-656.

10. Hamilton, V. L. (1978a). Who is responsible? Toward a social psychology of responsibility attribution. Social Psychology, 41, 316-328. http://dx.doi.org/10.2307/3033584

11. Hamilton, V. L. (1978b). Obedience and responsibility: A jury simulation. Journal of Personality and Social Psychology, 36, 126-146. http://dx.doi.org/10.1037/0022$\underline{3514.36 .2 .126}$

12. Hamilton, V. L. (1986). Chains of command: Responsibility attribution in hierarchies. Journal of Applied Social Psychology, 16, 118-138. http://dx.doi. org/10.1111/j.1559-1816.1986.tb02283.x

13. Hart, H. L. y Honoré, A. M. (1985). Causation in the law. Oxford, United Kingdom: Oxford University Press. http:// dx.doi.org/10.1093/acprof:oso/9780198254744.001.0001

14. Haynes, G.A. y Olson, J. M. (2006). Coping with threats to just-world beliefs: derogate, blame or help? Journal of Applied Social Psychology, 36, 664-682. http://dx.doi. org/10.1111/j.0021-9029.2006.00023.x

15. Heise, L. (1998). Violence against women. An integrated, ecological framework. Violence Against Women, 4, 262290. http://dx.doi.org/10.1177/1077801298004003002

16. Herrera, M. C., Expósito, F. Moya, M y Houston, D. (2012). "Having it all": Women's perception of impact of female promotion on threat of domestic violence. The Spanish Journal of Psychology, 15, 670-679.

17. Hoerl, K. (2002). Monstrous youth in suburbia: Disruption and recovery of the American dream. Southern Comunication Journal, 67, 259-275. http://dx.doi. org/10.1080/10417940209373235

18. King, N. (2010). Review of women, violence and the media: readings in feminist criminology. Gender and society, 24, 842-843. http://dx.doi.org/10.1177/0891243210369663

19. Koss, M. (2000). Blame, shame, and community: justice responses to violence against women. American Psychologist, 55, 1332-1343. http://dx.doi.org/10.1037/0003$\underline{066 X .55 .11 .1332}$

20. Lagnado, D. A. y Channon, S. (2008). Judgments of cause and blame: The effects of intentionality and foreseeability. Cognition, 108, 754-770. http://dx.doi.org/10.1016/j.cognition.2008.06.009

21. Leal González, D. A. y Arconada Melero, M. A. (2011).
Convivir en igualdad: prevención de violencia masculina hacia mujeres en todas las etapas educativas. Madrid: Universidad Nacional de Educación a Distancia.

22. Lerner, M. (1980). The belief in a just world: a fundamental delusion. New York: Plenum.

23. Lickel, B., Schmader, T. y Hamilton, D. L. (2003). A case of collective responsibility: Who else was to blame for the columbine High School Shootings? Personality and Social Psychology Bulletin, 29, 194-204. http://dx.doi. org $/ 10.1177 / 0146167202239045$

24. Macrae, C. N. y Milne, A. B. (1992). A curry for your thoughts: Empathic effects on counterfactual thinking. Personality and Social Psychology Bulletin, 18, 625-630. http://dx.doi.org/10.1177/0146167292185013

25. Mandel, D. R. (2003). Judgment dissociation theory: an analysis of differences in causal, counterfactual, and covariational reasoning. Journal of Experimental Psychology: General, 132, 419-434. http://dx.doi.org/10.1037/0096$\underline{3445.132 .3 .419}$

26. Mandel, D. R. y Lehman, D. R. (1996). Counterfactual thinking and ascriptions of cause and preventability. Journal of Personality and Social Psychology, 71, 450-63. http:// dx.doi.org/10.1037/0022-3514.71.3.450

27. Markman, K. D. y Tetlock, P.E. (2000). "I couldn't have known": Accountability, foreseeability and counterfactual denials of responsibility. The British Journal of Social Psychology, 39, 313-25. http://dx.doi. org/10.1348/014466600164499

28. McEleney, A. y Byrne, R. M. J. (2006). Spontaneous counterfactual thoughts and causal explanations. Thinking and Reasoning, 12, 235-255. http://dx.doi. org/10.1080/13546780500317897

29. McGill, A. L.y Klein, J. G. (1993). Contrastive and counterfactual thinking in causal judgement. Journal of Personality and Social Psychology, 64, 897-905. http://dx.doi. org/10.1037/0022-3514.64.6.897

30. Meindl, J. R., Ehrlich, S. B. y Dukerich, J. M. (1985). The romance of leadership. Administrative Science Quarterly, 30, 78-102. http://dx.doi.org/10.2307/2392813

31. Miller, D. T. y Gunasegaram, S. (1990). Temporal order and the perceived mutability of events: implications for blame assignment. Journal of Personality and Social Psychology, 59, 1111-118. http://dx.doi.org/10.1037/00223514.59.6.1111

32. Robbennolt, J. K. (2000). Outcome severity and judgments of "responsibility": a meta-analytic review. Journal of applied social psychology, 30, 2575-2609. http://dx.doi. org/10.1111/j.1559-1816.2000.tb02451.x

33. Roese, N. J. y Olson, J. M. (1995). Counterfactual thinking: A critical overview. En N. J. Roese y J. M. Olson (Eds.), What might have been: The social psychology of counterfactual thinking (pp. 1-59). Mahwah, NJ: Lawrence Erlbaum Associates. 
34. Roese, N. J. y Summervile, A. (2005). What we regret most... and why. Personality and Social Psychology Bulletin, 31, 1273-1285. http://dx.doi.org/10.1177/0146167205274693

35. Segura, S. y Morris, M. W. (2005). Scenarios simulations in learning: forms and functions at the individual and organizational levels. En D.R. Mandel, D. J. Hilton y P. Catellani (Eds.), The psychology of counterfactual thinking (pp. 94-109). New York: Routledge.

36. Shaver, K. G. (1985). The attribution of blame. Causality, responsibility, and blameworthiness. New York: SpringerVerlag. http://dx.doi.org/10.1007/978-1-4612-5094-4

37. Schlenker, B. R., Britt, T. W., Pennington, J., Murphy, R. y Doherty, K. (1994). The triangle model of responsibility. Psychological review, 101, 632-652. http://dx.doi. org/10.1037/0033-295X.101.4.632

38. Shultz, T., Schleifer, M. y Altman, I. (1981). Judgments of causation, responsibility, and punishment in cases of harmdoing. Canadian Journal of Behavioural Science 13, 238253. http://dx.doi.org/10.1037/h0081183

39. Shultz, T. R., Jaggi, C. y Scheleifer, M. (1987). Assigning vicarious responsibility. European Journal of Social Psychology, 17, 377-380. $\quad$ http://dx.doi.org/10.1002/ ejsp.2420170314

40. Stewart, A. y Maddren, K. (1997). Police officers' judgements of blame in family violence: The impact of gender and alcohol. Sex roles, 37, 921-933. http://dx.doi. org/10.1007/BF02936347

41. Thapar-Björkert, S. y Morgan, K. (2010). "But sometimes I think...they put themselves in the situation": exploring blame and responsibility in interpersonal violence. Violence against women, 16, 32-59. http://dx.doi. org/10.1177/1077801209354374

42. Valor-Segura, I., Expósito, F. y Moya, M. (2008).Atribución del comportamiento del agresor y consejo a la víctima en un caso de violencia doméstica. Revista de psicología social, 23, 171-180. http://dx.doi.org/10.1174/021347408784135896
43. Valor-Segura, I., Expósito, F. y Moya, M. (2011). Victim blaming and exoneration of the perpetrator in domestic violence: the role of beliefs in a just world and ambivalent sexism. The Spanish Journal of Psychology, 14, 195-206. http://dx.doi.org/10.5209/rev_SJOP.2011.v14.n1.17

44. Van Den Bos, K. y Mass, M. (2009). On the psychology of the belief in a just world: exploring experiential and rationalistic path to victim blaming. Personality and Social psychology Bulletin, 35, 1567-1578. http://dx.doi. org/10.1177/0146167209344628

45. Willis, G. B., Tapia-V, A. y Martínez, R. (2011). I control therefore I am: Effects of Mortality salience on control attributions. The Spanish Journal of Psychology, 14, 765-772.

46. Zhang, H. Zhou, L. y Luo, Y. (2009). The influence on the regret intensity: An ERP study. Acta Psicologica Sinica, 41, 454-463. http://dx.doi.org/10.3724/SP.J.1041.2009.00454

47. Zemba, Y., Young, M. J. y Morris, M. W. (2006). Blaming leaders for organizational accidents: Proxy logic in collective-versus individual-agency cultures. Organizational Behaviour and Human Decision Processes, 101, 36-51. http://dx.doi.org/10.1016/j.obhdp.2006.04.007

48. Zeelenberg, M., Van Dijk, W.W. y Manstead, S. R. (1998). Reconsidering the relation between regret and responsibility. Organizational Behaviour and Human Decision Processes, 74, 254-272. http://dx.doi.org/10.1006/ obhd.1998.2780

Fecha de recepción: 1 de julio de 2012 Fecha de recepción de la versión modificada: 9 de octubre de 2012 Fecha de aceptación: 7 de noviembre de 2012 